\title{
Kyste osseux anévrysmal bimaxillaire : présentation d'un cas
}

\author{
Suzy Flore Assini Eyogho ${ }^{1,2, \star}$, Jérôme Miloundja ${ }^{1,2}$, Paul Niang ${ }^{3}$, Sylvestre Mbamendame ${ }^{4}$, \\ François Ondo Ndong ${ }^{4}$, Boubacar Diallo ${ }^{3}$, Léon Nzouba ${ }^{1}$ \\ 1 Département de Chirurgie et Spécialités chirurgicales, Hôpital d'Instruction des Armées, Libreville, Gabon \\ 2 Départements d'ORL-Stomatologie et des Sciences morphologiques, Faculté de Médecine, Université des Sciences de la Santé, \\ Libreville, Gabon \\ 3 Département d'Odontologie, FMPOS-UCAD, Dakar, Sénégal \\ 4 Service de Chirurgie thoracique et vasculaire, Fondation Jeanne Ebori, Libreville, Gabon
}

(Reçu le 17 octobre 2010, accepté le 16 novembre 2010)

Mots clés :

kyste anévrysmal / maxillaire / exérèse
Résumé - Le kyste osseux anévrysmal (KOA) est une tumeur bénigne primitive ou secondaire, constituée par une cavité intra-osseuse, uni ou multiloculaire, à contenu hématique. Il peut se développer sur tous les os du squelette avec, cependant, une prédilection pour les os longs et le rachis. Le KOA touche principalement les enfants et les jeunes adultes, avec une légère prédominance féminine. Le but de la présentation de ce cas était de relever les particularités diagnostique, thérapeutique et pronostique de cette lésion qui lyse l'os de façon agressive et qui peut simuler une tumeur.

Nous rapportons un cas de KOA intéressant les deux maxillaires avec extension au sinus, découvert chez une patiente de 63 ans, survenu 10 ans après un traumatisme maxillo-facial. La patiente a bénéficié d'une exérèse large. L'évolution postopératoire a été favorable et, 9 mois après l'intervention, il n'y avait aucun signe clinique ou radiologique de récidive.

Pour la pathogénie du KOA il existe trois hypothèses : origine traumatique, vasculaire ou tumorale primaire. Plusieurs examens complémentaires permettent d'évoquer le diagnostic de K0A. L'examen anatomopathologique qui peut présenter des difficultés, reste le seul examen fiable. Le traitement de choix est la résection large avec reconstruction immédiate si la stabilité osseuse est compromise.

Le KOA peut être observé à tout âge et son pronostic reste globalement bon. Le bilan par imagerie est conseillé avant de réaliser une biopsie qui reste indispensable pour la confirmation du diagnostic.
Key words: aneurysmal cyst / jaw bones / resection
Abstract - Aneurysmal bone cyst involving both the two maxillaries: report of a case. Aneurysmal bone cyst $(A B C)$ is a benign tumour which can be primary or secondary carries out an osseous cavitation, one or multilocular, with hematic content. It can develop on all the bones of the skeleton with however a predilection for the long bones and the rachis. The $A B C$ concern mainly the children and young adults with a light female prevalence. The study intention here was to raise the diagnostic, therapeutic and prognostic particularities of this lesion which destroy the bone in an aggressive way and which can mimic other tumours clinical shape.

We report a case of anévrysmal cyst of two jawbones with sinusal extension, discovered in a 63 years old woman and maxillofacial traumatism notion which has occurred 10 years earlier. The patient has benefited from a wide tumoral resection. The postoperative development is marked, 9 months later, by a stable condition on clinic and radiological plans.

The pathogenesis of the $A B C$ is based on three theories: traumatic, vascular and tumoral. Several complementary examinations make mention $\mathrm{ABC}$ diagnosis. The histopathological examination which can present shelves, remains the only certainty examination. The best treatment is wide résection with immediate rebuilding if stability is compromised.

The $A B C$ can be observed at any age and its pronoctic is generally right. The modern X-ray contribution become essential but the biopsy remains more reliable.

\footnotetext{
^Correspondance : eyoghoass@yahoo.fr
} 
Selon la définition de l'0MS (2002), le kyste osseux anévrysmal (KOA) est une lésion kystique bénigne des os, constituée de cavités remplies de sang, séparées les unes des autres par des septum de tissu conjonctif comportant des fibroblastes, des cellules géantes ostéoclastiques et un os trabéculaire réactionnel plus ou moins abondant [1]. Le KOA a été décrit pour la première fois en 1942 par Jaffe et Lichtenstein.

Histologiquement bénin, le KOA peut cependant avoir une évolution agressive : risque de fracture majeure lorsque l'ostéolyse est importante, fréquence des récidives locales et parfois séquelles sévères [2]. En principe, la résection large avec reconstruction immédiate constitue le traitement de choix; toutefois, la localisation du KOA, son volume et son degré d'agressivité déterminent l'attitude chirurgicale pour la résection, comme pour la reconstruction, s'il existe une zone de faiblesse mécanique [3].

Le cas rapporté intéresse les deux maxillaires avec extension dans le sinus maxillaire [4] chez une patiente de 63 ans.

\section{Observation}

Mme LP, de nationalité gabonaise, femme au foyer, a consulté le 15/04/2009 pour des douleurs maxillofaciales diffuses, d'évolution progressive, avec des paroxysmes nocturnes et un écoulement liquidien intermittent dans la cavité buccale; la symptomatologie évoluait depuis plus de 3 ans.

A l'examen exobuccal, on notait une discrète tuméfaction latéro-mandibulaire bilatérale, faisant corps avec l'os, associée à un léger trismus. L’hygiène bucco-dentaire était correcte et il n'y avait pas d'adénopathies cervicofaciales. La palpation de la branche montante droite de la mandibule et de la région narinaire gauche était douloureuse.

L'examen endobuccal montrait des tuméfactions latérales, intéressant les deux maxillaires saillant dans les vestibules; la muqueuse de surface avait par endroits une couleur bleutée et semblait amincie. Les tissus mous étaient légèrement refoulés. La denture était complète (32 dents) sans lésion associée (Fig. 1). Le reste de l'examen clinique était normal. La radiographie panoramique montrait une lyse osseuse bilatérale, touchant les branches montantes et les branches horizontales, mais respectant la région symphysaire (Fig. 2). Le CT-scan du massif facial (Fig. 3a, 3b et 3c) montraient des images kystiques multiloculaires avec une extension dans le sinus maxillaire gauche associée à une dent incluse et une sinusite maxillaire et des images kystiques dans la mandibule.

L'anamnèse de la patiente révélait un traumatisme maxillofacial violent, survenu au cours d'une dispute conjugale il y a un peu plus de 10 ans; la patiente avait reçu en pleine face une lampe torche.

Elle a été hospitalisée, du 2 au 13 juin 2009, dans le service d'Odontostomatologie et Chirurgie maxillofaciale de l'Hôpital d'Instruction des Armées Omar Bongo-Ondimba et, le 8 juin 2009, une exérèse large de la tumeur a été réalisée. Après le décollement mucopériosté, on a découvert des corticales maxillaire et mandibulaire soufflées (Fig. 4). L'ouverture des

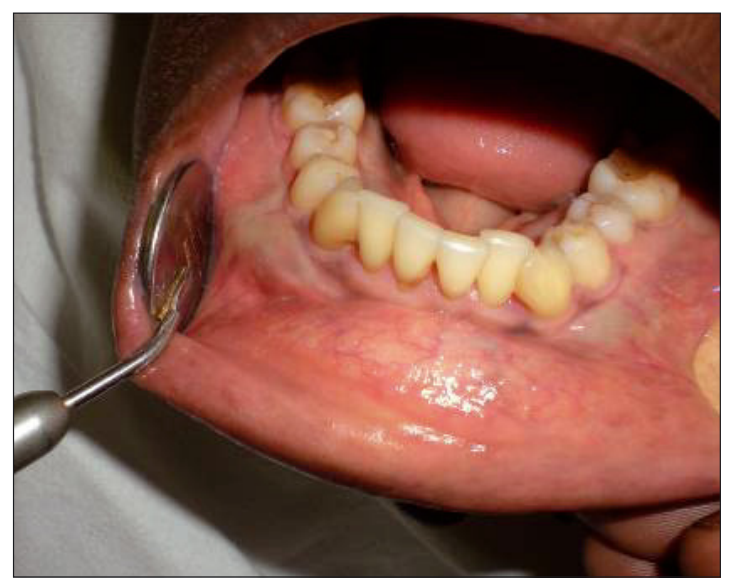

Fig. 1. Tuméfaction latéro-mandibulaire bleuâtre, comblant en partie le vestibule, qui semble due à une soufflure de la corticale.

Fig. 1. Mandibular curve filling the hall and letting show through cortical puffed up bluish.

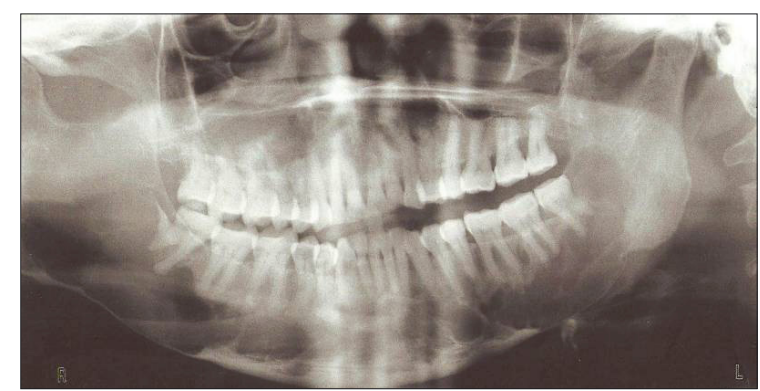

Fig. 2. Radiographie panoramique montrant une lésion ostéolytique bilatérale des branches horizontales et montantes de la mandibule.

Fig. 2. Panoramic $x$-ray showing an osteolytic lesion in horizontal and vertical branches of mandibular.

cavités kystiques a libéré un liquide rouge brunâtre, hématique, non coagulé ; l'aspiration à l'aiguille a ramené environ 15 cc de liquide des cavités mandibulaires et 5 cc des cavités maxillaires (Fig. 5). Ensuite, il a été procédé à l'exérèse de toutes les corticales osseuses externes atteintes (Fig. 6a et $6 \mathrm{~b})$. Lors de cette décortication, on a conservé les corticales internes, la lamina dura des alvéoles dentaires et le nerf alvéolaire inférieur qui était libre dans la cavité (Fig. 7). Après le curetage, un méchage et un drainage ont été placés dans les cavités. L'examen anatomopathologique du produit de curetage a conclu à un KOA. Neuf mois plus tard, l'évolution clinique et radiologique était satisfaisante : le CT-scan (Fig. 8a et 8b) montrait l'absence de lésion osseuse et les sinus maxillaires avaient une radiotransparence normale. Il persistait toutefois une symptomatologie douloureuse dans les maxillaires, mais de faible intensité.

\section{Discussion}

Le KOA est une lésion dystrophique rare du squelette qui représente $6 \%$ de l'ensemble des tumeurs osseuses. Il touche 


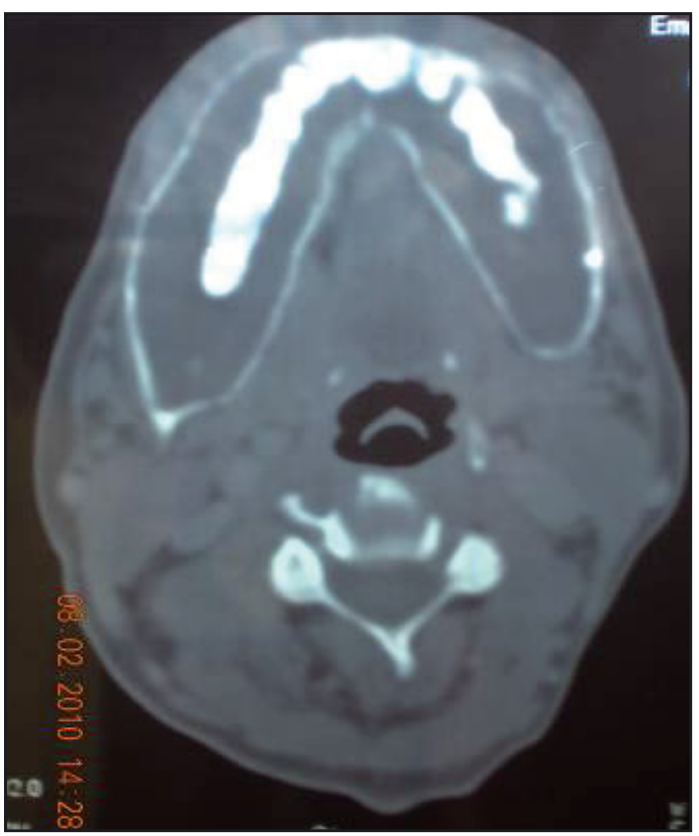

(a)

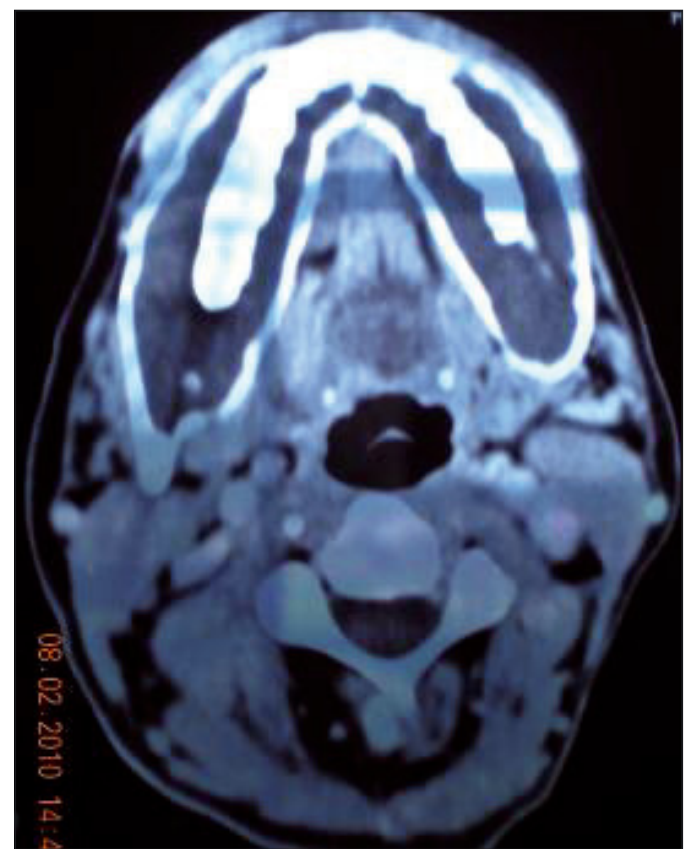

(b)

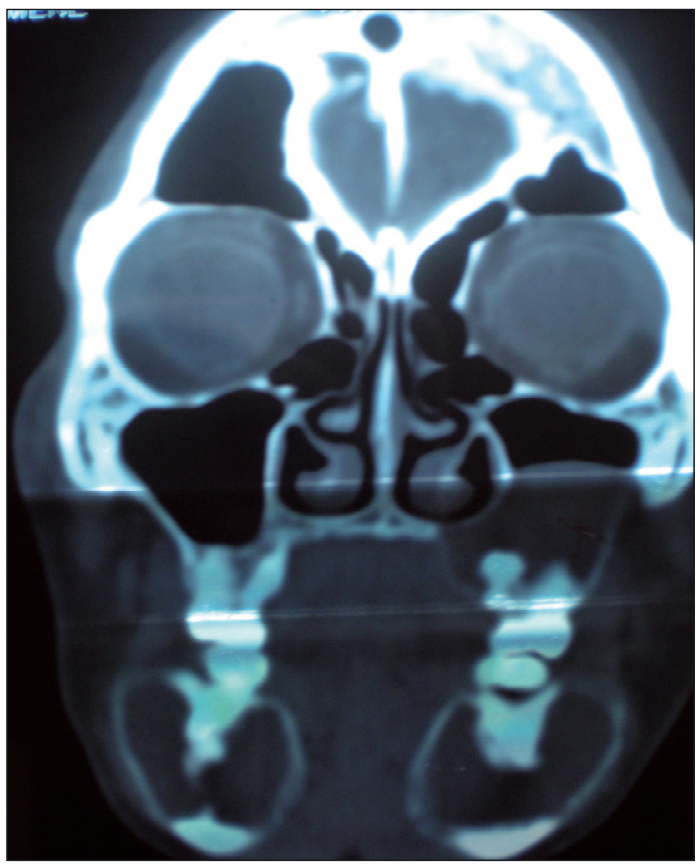

(c)

Fig. 3. (a et b) CT-scan montrant une lésion ostéolytique bien délimitée, d'aspect kystique, soufflant les corticales de la mandibule sauf dans la région symphysaire (coupe axiale) : (a) en fenêtre tissus mous, (b) en fenêtre osseuse ; (c) CT-scan montrant des images kystique dans la mandibule et le maxillaire avec extension dans le sinus maxillaire gauche (coupe coronale).

Fig. 3. ( $a$ and b) axial CT-scan showing a well délimited ostolytic lesion of cystic, blowing aspect the corticals of the mandibule, excepted in the median region using (a) soft tissue algorithm, (b) bone algorithm; (c) coronal CT-scan showing the kystic image in the jaw bones with left sinusal extension. 


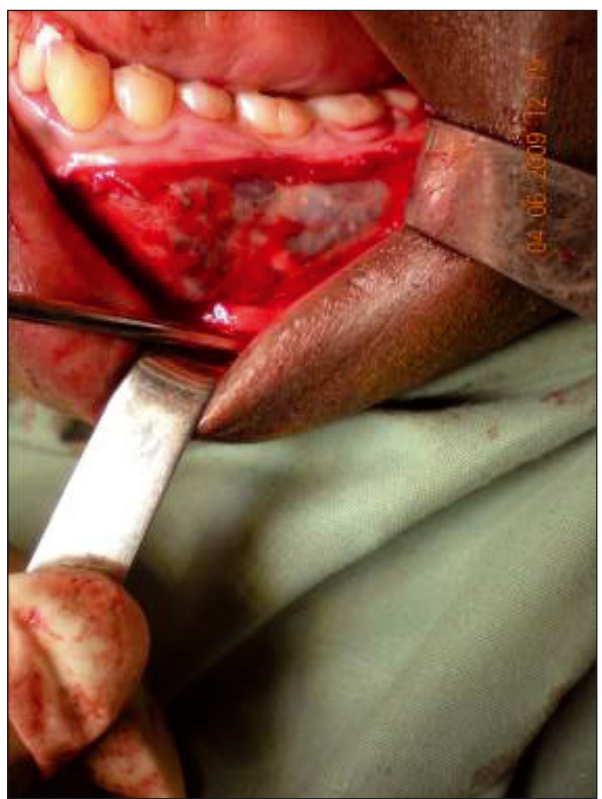

Fig. 4. Corticale mandibulaire soufflée.

Fig. 4. Puffed up mandibular bone.

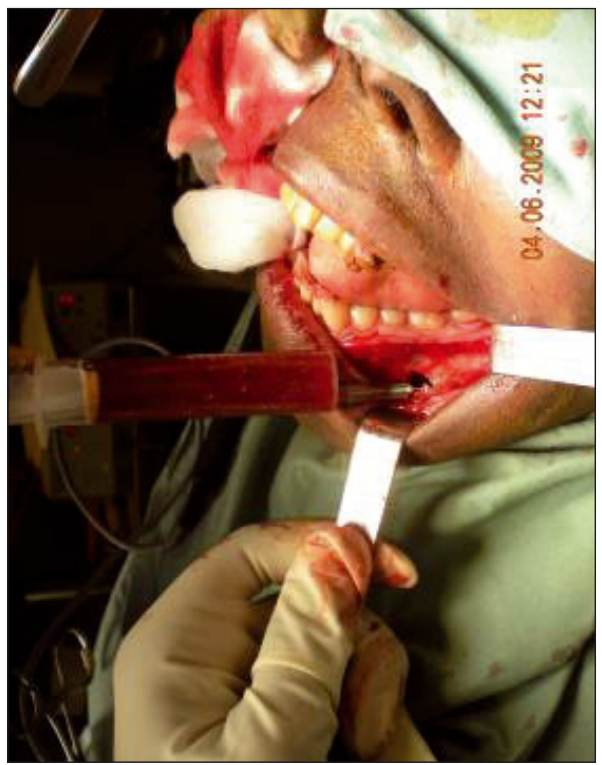

Fig. 5. Aspiration à l'aiguille du contenu de la cavité kystique mandibulaire.

Fig. 5. Cystic punction.

principalement les enfants et les jeunes adultes, avec une discrète prédominance féminine [5]. Entité lésionnelle isolée dans $70 \%$ des cas, il peut accompagner une autre lésion qu'il faut systématiquement rechercher [5]. Le KOA mandibulaire reste très rare et sa localisation maxillaire est exceptionnelle [6]. A la mandibule, il atteint de manière préférentielle les régions molaire et angulaire et la branche montante [7] ; la région symphysaire est généralement respectée, comme dans le cas rapporté.

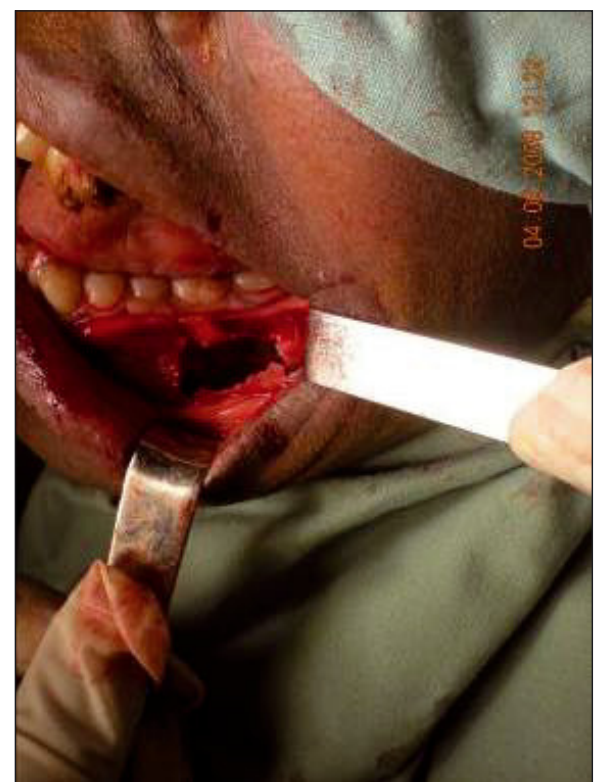

(a)

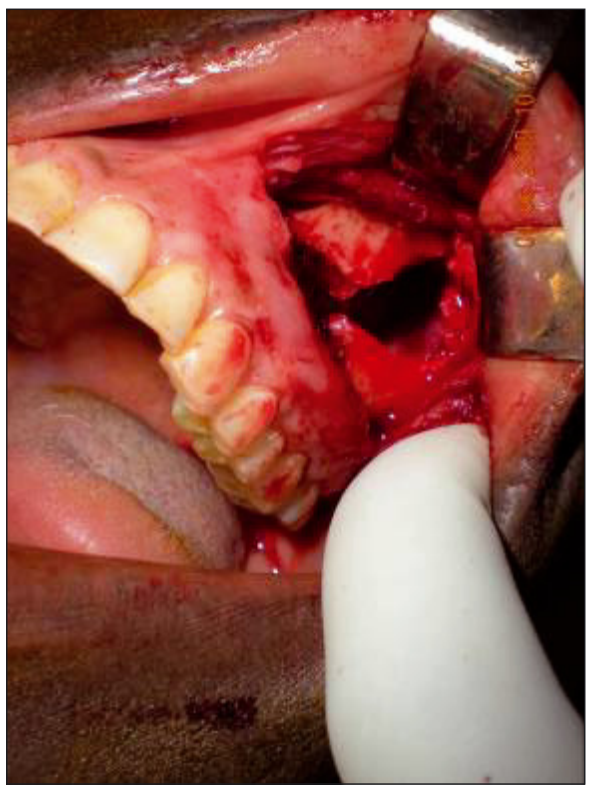

(b)

Fig. 6. (a) Décortication osseuse à la mandibule. (b) Décortication osseuse au maxillaire montrant l'extension dans le sinus maxillaire gauche.

Fig. 6. (a) Osseous mandibular hulling. (b) Maxillar hulling showing left sinusal extension.

Le KOA se manifeste le plus souvent par des douleurs [8] ; elles ont constitué le principal signe d'appel dans le cas rapporté. Dans un tiers des cas, le KOA qui comporte une agressivité locale, entraine un remaniement des corticales qui sont soufflées et amincies; le KOA peut être à l'origine d'une fracture pathologique. Il existe des KOA dits solides : dans ces cas, il existe une ostéogénèse réactionnelle qui donne des 


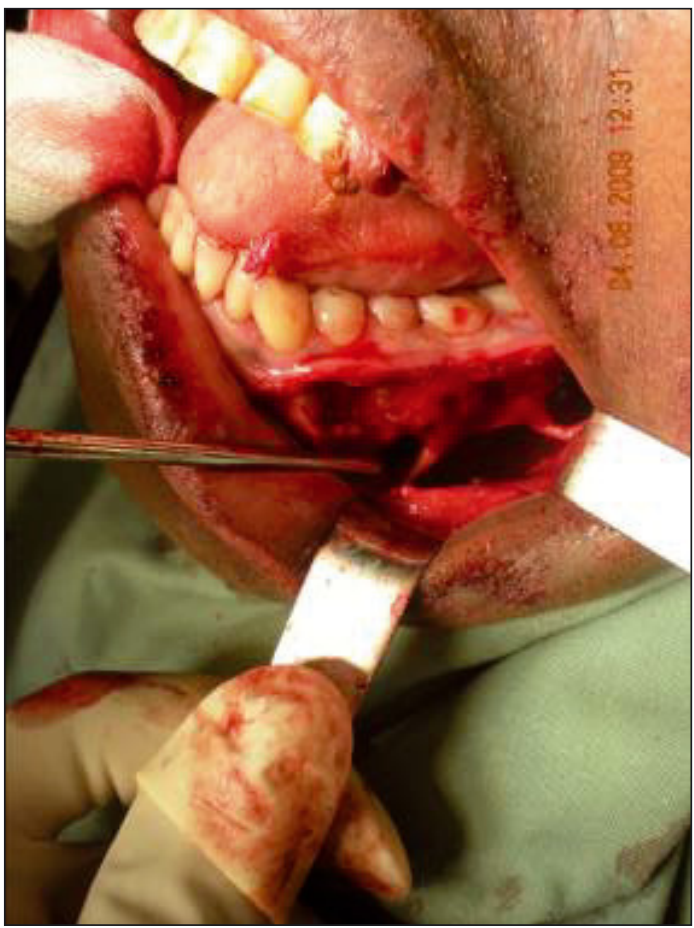

Fig. 7. Nerf alvéolaire inférieur libre dans la cavité.

Fig. 7. Free alveolar nerve in the cavity.

travées de substance ostéoïde avec un degré de calcification variable : ceci expliquerait leur bon pronostic et la possibilité de guérison sans récidive dans certains cas, même lorsque l'exérèse n'a pas été complète [9].

Le KOA est souvent comparé à une éponge dont les cavités sont remplies de sang. Ce n'est pas une vraie tumeur, ni un kyste, ni un véritable anévrysme, mais il correspond vraisemblablement à un processus réactionnel [10]. Il constitue un pseudo-kyste et son pronostic reste globalement bon, même si quelques cas de transformations malignes ont été décrits [8].

Pour Jaffe et Lichtenstein [1], le KOA représente une lésion osseuse réactionnelle, secondaire à une perturbation hémodynamique due à une augmentation de la pression veineuse consécutive à une thrombose ou à une communication artérioveineuse anormale. Bieseker et al. ont émis l'hypothèse que le KOA serait secondaire à une fistule artérioveineuse préexistante : l'hyperpression qui en résulte, entraînerait une résorption osseuse [3]. Pour Campanacci et Ruggieri, il s'agirait d'une réparation tissulaire après une hémorragie locale, ce qui expliquerait le développement d'un KOA après fracture sur un os sain ou pathologique [11]. Pour Kind, la forme sous-périostée surviendrait plutôt après une lésion post-traumatique. La notion de traumatisme maxillofacial direct retrouvée dans notre observation semble confirmer les hypothèses décrites ci-dessus; plus particulièrement celle d'un hématome sous-périosté qui pourrait être à l'origine du KOA dans le cas rapporté.

Le CT-scan et l'IRM apportent des éléments précieux pour le diagnostic du KOA mais ils doivent être bien conduits afin de ne pas méconnaître le $K O A$; dans le cas rapporté, un

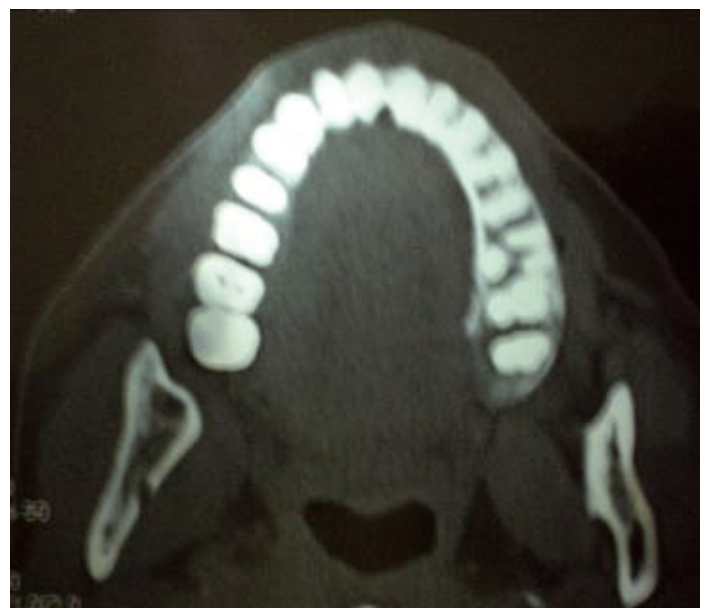

(a)

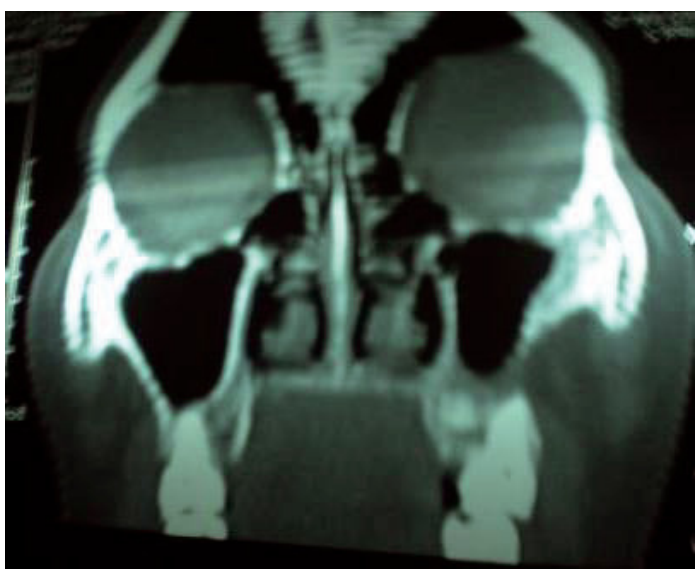

(b)

Fig. 8. (a) CT-scan réalisé 9 mois après l'intervention chirurgicale : disparition de la cavité kystique mandibulaire. (b) CT-scan réalisé 9 mois après l'intervention chirurgicale : les sinus maxillaires ont retrouvé une radiotransparence normale.

Fig. 8. (a) CT-scan 9 months after surgery: mandibular cystic cavity has disappeared. (b) CT-scan 9 months after surgery: sinus clear image

examen trop rapide avait conclu initialement à un kératokyste. Pour plusieurs auteurs [11], la biopsie est beaucoup plus fiable que la ponction pour confirmer le diagnostic.

Pour le traitement [11], la radiothérapie a été abandonnée; elle reste utilisée pour les KOA au-dessus de toute possibilité d'exérèse chirurgicale. L'injection intralésionnelle de corticoïdes et l'implantation de particules osseuses partiellement déminéralisées pourraient faire évoluer le KOA de la phase de résorption ostéolytique vers celle de reconstruction, évitant ainsi une chirurgie invasive. La transfixion par des broches de Kirschner et l'injection intrakystique d'éthibloc représentent des modalités thérapeutiques complémentaires peu utilisées. L'embolisation est indiquée en préopératoire pour les lésions très étendues afin d'éviter un saignement important. Le traitement purement chirurgical consiste en un 
curetage simple du KOA, avec ou sans greffe osseuse; la fréquence des récidives est de l'ordre de 20 à $40 \%$.

Pour les maxillaires, le traitement repose le plus souvent sur un simple curetage. La récidive est rare à la mandibule et apparaît en général dans les 30 mois qui suivent l'exérèse chirurgicale [7]. C'est une attitude thérapeutique qui a été adoptée pour le cas rapporté, le plateau technique ne permettant guère d'envisager une greffe osseuse ou le recours à des matériaux de comblement. Après 9 mois, l'évolution semblait favorable et il n'y avait aucun signe de récidive.

\section{Conclusion}

Le KOA, lésion d'aspect souvent impressionnant à l'imagerie, peut être observée à tout âge et son pronostic reste globalement bon. L'imagerie constitue un examen complémentaire indispensable. Le CT-scan et l'IRM permettent d'analyser les limites corticales et extracorticales du KOA. La biopsie représente un temps indispensable pour le diagnostic. Le traitement de choix est la résection de la lésion avec exérèse large, suivie d'une reconstruction immédiate lorsque la stabilité est compromise. Quelle que soit l'option thérapeutique, il importe d'éviter tout sacrifice fonctionnel, puisqu'il s'agit d'une lésion de nature bénigne, donc potentiellement curable avec des moyens conservateurs.

\section{Conflits d'intérêt : aucun}

\section{Références}

1. Jaffe HL, Lichtenstein L. Solitary unicameral bone cysts : with emphasis on the roentgen picture, the pathologic appearance and the pathogenesis. Arch Surg 1942;44:1004-25.
2. Bollini G, Panuel $M$, Jouve JL, Scheiner C, Toth C, Lemaire $P$, Jacquemier M. Kyste anévrysmal (pp. 157-72). In Les tumeurs osseuses de l'enfant. Lascombes $P$, Lefort $G$ ed. Monographies du GEOP, Montpellier, Sauramps médical, 1996.

3. Biesecker JL, Marcove RC, Huvos AG, Mike V. Aneurysmal bone cysts: a clinicopathologic study of 66 cases. Cancer 1970;26:615-25.

4. Mazabraud A. Kyste anévrismatique (pp. 335-52). In Anatomie pathologique osseuse tumorale. Springer-Verlag, Paris, 1994.

5. Albiñana J, González-Morán G, Morcuende JA. Femoral avascular necrosis associated with metaphyseal aneurysmal bone cyst. J Pediatric Orthop (part B) 1995;4:110-3.

6. Ginisty D, Mettoudi JD, Adamsbaum C, Dhellemmes C, Maillet S. Tumeurs bénignes et pseudotumeurs des maxillaires de l'enfant. Rev Stomatol Chir Maxillofac 1996;97:12-6.

7. Jeblaoui Y, Ben Neji N, Haddad S, Zribi A, Néji R, Boussafa $H$, Sahtout S, Hchicha S. Kyste osseux anévrismal mandibulaire mimant une tumeur maligne. Rev Stomatol Chir Maxillofac 2007;108:238-40.

8. Dutoit M, Kaelin A, Jundt G, Siebenrock K, von Hochstetter A, Hefti F. Kyste osseux anévrysmal (KOA). Forum médical, symposium 2007;7:371-4.

9. Sanerkin NG, Mott MG, Roulance J. An unusual intraosseus lésion with fibroblastic, osteoclastic, osteoblastic, aneurismal and fibromixoid elements: "solid" variant of aneurismal bone cyst. Cancer 1983;51:2278-86.

10. Vergel de Dios AM, Bond JR, Schives TC, McLeod RA, Krishnan Unni K. Aneurysmal bone cyst: a clinicopathologic study of 238 cases. Cancer, 1992;69:2921-31.

11. Cottalorda J, Bollini G, Panuel M, Scheiner C, Jouve JL, Labriet C, Bouyala JM. Le kyste anévrismal des os chez l'enfant. Rev Chir Orthop 1993;79:272-80.

12. Campanacci M, Ruggieri P. Lésions pseudotumorales: kyste anévrismal. Encycl Med Chir Appareil locomoteur, 14-030-K-10. Elsevier-Masson, Paris, 1993. 